Supplement of The Cryosphere, 12, 3853-3859, 2018

https://doi.org/10.5194/tc-12-3853-2018-supplement

(C) Author(s) 2018. This work is distributed under

the Creative Commons Attribution 4.0 License.

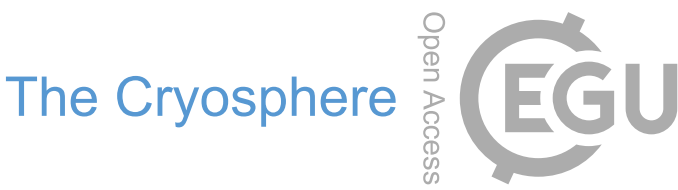

(c) (1)

Supplement of

\title{
Brief communication: widespread potential for seawater infiltration on Antarctic ice shelves
}

Sue Cook et al.

Correspondence to: Sue Cook (sue.cook@utas.edu.au)

The copyright of individual parts of the supplement might differ from the CC BY 4.0 License. 


\begin{tabular}{|c|c|c|c|c|c|c|c|}
\hline Ice Shelf & Bedmap2 & CryoSat-2 & GLAS & RAMP & REMA & Mean & STD \\
\hline Abbot & 45.0 & 47.1 & 50.0 & 52.1 & 47.6 & 48.4 & 2.7 \\
\hline Amery & 0.4 & 1.7 & 7.5 & 8.0 & 2.0 & 3.9 & 3.6 \\
\hline Bach & 15.5 & 22.5 & 27.6 & 25.4 & 19.5 & 22.1 & 4.8 \\
\hline Cook & 4.2 & 1.5 & 0.0 & 38.0 & 10.9 & 10.9 & 15.7 \\
\hline Crosson & 60.0 & 69.4 & 74.3 & 66.9 & 79.6 & 70.0 & 7.4 \\
\hline Dibble & 1.7 & 9.5 & 25.7 & 20.7 & 9.4 & 13.4 & 9.7 \\
\hline Dotson & 14.0 & 24.0 & 24.6 & 15.3 & 24.4 & 20.5 & 5.3 \\
\hline Drygalski & 20.1 & 17.4 & 0.0 & 37.2 & 23.4 & 19.6 & 13.3 \\
\hline Edward VIII Bay & 17.3 & 10.6 & 21.8 & 29.5 & 24.3 & 20.7 & 7.1 \\
\hline Filchner-Ronne & 0.8 & 0.4 & 1.9 & 1.1 & 0.3 & 0.9 & 0.7 \\
\hline George VI & 29.3 & 33.0 & 34.7 & 41.1 & 36.0 & 34.8 & 4.3 \\
\hline Getz & 8.2 & 11.2 & 16.3 & 15.8 & 14.5 & 13.2 & 3.4 \\
\hline Jelbartisen \& Fimbul & 7.3 & 6.9 & 17.3 & 12.6 & 8.3 & 10.5 & 4.4 \\
\hline Larsen C-D & 21.9 & 20.9 & 22.8 & 29.0 & 25.5 & 24.0 & 3.3 \\
\hline Mertz & 3.8 & 5.2 & 0.2 & 13.3 & 7.1 & 5.9 & 4.8 \\
\hline Moscow Un & 0.1 & 1.0 & 2.0 & 1.9 & 0.6 & 1.1 & 0.8 \\
\hline Nickerson & 45.2 & 45.7 & 0.8 & 54.3 & 49.7 & 39.1 & 21.7 \\
\hline Pine Island & 7.5 & 10.4 & 20.3 & 18.6 & 17.3 & 14.8 & 5.5 \\
\hline Prince Harald & 55.4 & 50.8 & 65.0 & 68.5 & 55.6 & 59.0 & 7.4 \\
\hline Princess Ragnhild & 16.2 & 15.9 & 26.8 & 24.2 & 15.9 & 19.8 & 5.3 \\
\hline Rennick & 9.7 & 9.2 & 0.0 & 30.0 & 17.3 & 13.3 & 11.2 \\
\hline Riiser-Larsen \& Brunt & 31.4 & 29.6 & 38.8 & 38.9 & 34.1 & 34.6 & 4.2 \\
\hline Ross & 2.5 & 2.4 & 0.1 & 2.6 & 2.8 & 2.1 & 1.1 \\
\hline Shackleton & 29.1 & 34.9 & 43.1 & 42.4 & 36.7 & 37.2 & 5.8 \\
\hline Sulzberger & 26.5 & 26.2 & 0.1 & 32.2 & 30.4 & 23.1 & 13.1 \\
\hline Thwaites & 3.5 & 25.1 & 53.7 & 30.0 & 50.4 & 32.5 & 20.5 \\
\hline Totten & 1.4 & 5.6 & 21.5 & 10.3 & 6.9 & 9.1 & 7.6 \\
\hline Venable & 15.0 & 60.7 & 72.6 & 53.0 & 68.2 & 53.9 & 23.0 \\
\hline West & 18.4 & 18.8 & 41.9 & 33.0 & 20.4 & 26.5 & 10.5 \\
\hline Wilkins & 69.6 & 82.0 & 73.2 & 88.0 & 73.8 & 77.3 & 7.5 \\
\hline All shelves & 8.7 & 9.0 & 11.0 & 12.3 & 10.1 & 10.2 & 1.5 \\
\hline
\end{tabular}

Table S1: Percentage ice shelf areas covered by $750 \mathrm{~kg} \mathrm{~m}^{-3}$ brine zone, using different surface DEMs as input. 


\begin{tabular}{|c|c|c|c|c|c|c|c|}
\hline Ice Shelf & Bedmap2 & CryoSat-2 & GLAS & RAMP & REMA & Mean & STD \\
\hline Abbot & 81.5 & 81.8 & 74.1 & 83.8 & 84.7 & 81.2 & 4.2 \\
\hline Amery & 10.1 & 10.3 & 13.8 & 15.0 & 11.6 & 12.2 & 2.2 \\
\hline Bach & 41.7 & 52.6 & 44.2 & 48.1 & 50.3 & 47.4 & 4.4 \\
\hline Cook & 17.7 & 10.5 & 7.0 & 42.7 & 28.2 & 21.2 & 14.5 \\
\hline Crosson & 85.0 & 83.9 & 83.0 & 85.5 & 88.7 & 85.2 & 2.2 \\
\hline Dibble & 19.1 & 22.7 & 53.5 & 38.8 & 27.6 & 32.3 & 14.0 \\
\hline Dotson & 42.7 & 54.1 & 55.2 & 40.8 & 54.5 & 49.5 & 7.1 \\
\hline Drygalski & 40.4 & 33.9 & 0.0 & 55.1 & 44.5 & 34.8 & 20.9 \\
\hline Edward VIII Bay & 41.1 & 28.2 & 32.8 & 44.4 & 43.0 & 37.9 & 7.1 \\
\hline Filchner-Ronne & 2.4 & 2.2 & 3.4 & 2.8 & 1.9 & 2.5 & 0.6 \\
\hline George VI & 50.4 & 54.8 & 53.0 & 63.6 & 56.9 & 55.7 & 5.0 \\
\hline Getz & 33.3 & 43.8 & 42.8 & 42.7 & 45.9 & 41.7 & 4.9 \\
\hline Jelbartisen \& Fimbul & 37.3 & 37.2 & 43.9 & 42.3 & 37.3 & 39.6 & 3.2 \\
\hline Larsen C-D & 35.7 & 34.9 & 36.0 & 43.9 & 40.3 & 38.2 & 3.9 \\
\hline Mertz & 23.1 & 18.9 & 25.9 & 29.1 & 28.0 & 25.0 & 4.1 \\
\hline Moscow University & 0.5 & 1.8 & 6.3 & 3.0 & 1.9 & 2.7 & 2.2 \\
\hline Nickerson & 72.1 & 70.4 & 31.2 & 77.4 & 74.7 & 65.2 & 19.2 \\
\hline Pine Island & 39.2 & 58.9 & 60.7 & 54.1 & 67.0 & 56.0 & 10.4 \\
\hline Prince Harald & 85.3 & 81.3 & 89.2 & 89.4 & 84.8 & 86.0 & 3.4 \\
\hline Princess Ragnhild & 40.5 & 41.3 & 49.0 & 46.3 & 40.1 & 43.5 & 4.0 \\
\hline Rennick & 28.0 & 26.5 & 0.0 & 43.1 & 36.1 & 26.8 & 16.4 \\
\hline Riiser-Larsen \& Brunt & 65.6 & 64.7 & 67.1 & 70.0 & 63.0 & 66.1 & 2.6 \\
\hline Ross & 12.1 & 12.1 & 6.1 & 12.1 & 11.8 & 10.8 & 2.6 \\
\hline Shackleton & 69.9 & 69.4 & 74.1 & 75.7 & 71.2 & 72.1 & 2.7 \\
\hline Sulzberger & 52.7 & 54.7 & 37.8 & 62.2 & 58.5 & 53.2 & 9.3 \\
\hline Thwaites & 45.9 & 53.6 & 81.1 & 63.3 & 84.8 & 65.7 & 16.9 \\
\hline Totten & 8.1 & 11.2 & 32.9 & 19.3 & 17.6 & 17.8 & 9.6 \\
\hline Venable & 93.9 & 93.5 & 94.5 & 86.2 & 99.0 & 93.4 & 4.6 \\
\hline West & 39.6 & 36.2 & 58.4 & 48.5 & 40.9 & 44.7 & 8.9 \\
\hline Wilkins & 94.1 & 94.6 & 85.9 & 98.8 & 96.6 & 94.0 & 4.9 \\
\hline Total & 21.7 & 22.0 & 21.3 & 24.4 & 22.6 & 22.4 & 1.2 \\
\hline
\end{tabular}

Table S2: Percentage ice shelf areas covered by $800 \mathrm{~kg} \mathrm{~m}^{-3}$ brine zone, using different surface DEMs as input. 


\begin{tabular}{|c|c|c|c|c|c|c|c|}
\hline Ice Shelf & Bedmap2 & CryoSat-2 & GLAS & RAMP & REMA & Mean & STD \\
\hline Abbot & 95.5 & 92.1 & 93.2 & 93.2 & 96.7 & 94.2 & 1.9 \\
\hline Amery & 20.2 & 20.7 & 24.0 & 24.0 & 21.5 & 22.1 & 1.8 \\
\hline Bach & 57.2 & 65.1 & 58.4 & 58.4 & 69.1 & 61.6 & 5.2 \\
\hline Cook & 35.5 & 23.2 & 46.5 & 46.5 & 45.9 & 39.5 & 10.3 \\
\hline Crosson & 89.6 & 90.8 & 89.2 & 89.2 & 93.2 & 90.4 & 1.7 \\
\hline Dibble & 28.6 & 31.0 & 49.0 & 48.1 & 40.1 & 39.4 & 9.4 \\
\hline Dotson & 72.1 & 79.5 & 60.8 & 60.8 & 81.3 & 70.9 & 9.9 \\
\hline Drygalski & 50.2 & 47.1 & 65.7 & 65.7 & 56.5 & 57.0 & 8.6 \\
\hline Edward VIII Bay & 62.1 & 42.2 & 58.6 & 58.6 & 63.7 & 57.0 & 8.6 \\
\hline Filchner-Ronne & 8.3 & 8.0 & 8.3 & 8.3 & 6.7 & 7.9 & 0.7 \\
\hline George VI & 73.9 & 78.2 & 82.3 & 82.3 & 81.2 & 79.6 & 3.6 \\
\hline Getz & 59.8 & 67.7 & 65.0 & 65.0 & 70.1 & 65.5 & 3.8 \\
\hline Jelbartisen \& Fimbul & 63.6 & 62.9 & 66.0 & 65.6 & 64.2 & 64.5 & 1.3 \\
\hline Larsen C-D & 49.5 & 48.8 & 58.8 & 58.8 & 53.0 & 53.8 & 4.9 \\
\hline Mertz & 42.1 & 34.1 & 49.7 & 49.7 & 43.1 & 43.7 & 6.4 \\
\hline Moscow University & 1.5 & 2.4 & 4.8 & 4.8 & 3.9 & 3.5 & 1.4 \\
\hline Nickerson & 82.8 & 80.6 & 94.7 & 94.7 & 86.1 & 87.8 & 6.6 \\
\hline Pine Island & 67.2 & 77.5 & 77.8 & 77.8 & 83.5 & 76.8 & 5.9 \\
\hline Prince Harald & 95.7 & 93.1 & 97.6 & 97.6 & 95.2 & 95.8 & 1.9 \\
\hline Princess Ragnhild & 60.9 & 62.5 & 68.1 & 68.1 & 60.8 & 64.1 & 3.7 \\
\hline Rennick & 39.3 & 35.7 & 49.5 & 49.5 & 44.8 & 43.8 & 6.1 \\
\hline Riiser-Larsen \& Brunt & 87.1 & 86.0 & 89.5 & 89.5 & 85.6 & 87.5 & 1.9 \\
\hline Ross & 40.3 & 43.5 & 40.1 & 40.1 & 40.7 & 41.0 & 1.4 \\
\hline Shackleton & 82.2 & 81.3 & 85.8 & 85.8 & 84.2 & 83.9 & 2.1 \\
\hline Sulzberger & 68.3 & 69.3 & 77.8 & 77.8 & 74.2 & 73.5 & 4.5 \\
\hline Thwaites & 80.7 & 82.8 & 88.0 & 88.0 & 95.4 & 87.0 & 5.7 \\
\hline Totten & 17.5 & 19.2 & 28.8 & 28.8 & 27.1 & 24.3 & 5.5 \\
\hline Venable & 96.8 & 95.7 & 89.3 & 89.3 & 99.6 & 94.2 & 4.6 \\
\hline West & 56.8 & 54.3 & 64.5 & 64.5 & 60.0 & 60.0 & 4.6 \\
\hline Wilkins & 96.0 & 96.4 & 99.2 & 99.2 & 98.1 & 97.8 & 1.5 \\
\hline Total & 39.3 & 40.4 & 41.2 & 41.2 & 40.1 & 40.5 & 1.0 \\
\hline
\end{tabular}

Table S3: Percentage ice shelf areas covered by $830 \mathrm{~kg} \mathrm{~m}^{-3}$ brine zone, using different surface DEMs as input. 


\begin{tabular}{|c|c|c|c|c|c|}
\hline Identifier & Shelf & Lat $\left(^{\circ}\right)$ & Lon $\left(^{\circ}\right)$ & Brine & Reference \\
\hline J-9 & Ross & -82.375 & -168.626 & $\mathrm{~N}$ & (Zotikov et al., 1980) \\
\hline J9 & Ross & -78.367 & 179.85 & $\mathrm{~N}$ & (Herron and Langway, 1979) \\
\hline T340 & Ronne & -79.4 & -64.5 & $\mathrm{~N}$ & (Graf et al., 1988) \\
\hline B04 & Ekströmisen & -70.62 & -8.367 & $\mathrm{~N}$ & (Schlosser and Oerter, 2002) \\
\hline B03 & Ekströmisen & -70.62 & -8.367 & $\mathrm{~N}$ & (Schlosser et al., 1999) \\
\hline FB0198 & Ekströmisen & -70.73 & -8.19 & $\mathrm{~N}$ & (Schlosser and Oerter, 2002) \\
\hline $\mathrm{B} 15$ & Filchner-Ronne & -77.935 & -55.9361 & $\mathrm{~N}$ & (Graf et al., 1994) \\
\hline B13 & Filchner-Ronne & -76.9811 & -52.2678 & $\mathrm{~N}$ & (Graf et al., 1994) \\
\hline Little America V & Ross & -78.167 & -162.22 & $\mathrm{~N}$ & (Gow, 1968) \\
\hline Lazarev & Lazarev & -69.967 & 12.92 & $\mathrm{Y}$ & (Dubrovin, 1960) \\
\hline $\begin{array}{l}\text { Base Roi } \\
\text { Baudouin }\end{array}$ & Roi-Baudouin & -70.4314 & 24.3106 & $\mathrm{~N}$ & (Tongiorgi et al., 1962) \\
\hline G1 & Amery & -69.451 & 71.497 & $\mathrm{~N}$ & (Budd et al., 1982) \\
\hline $\mathrm{C}-16$ & Ross & -81.1935 & 170.4978 & $\mathrm{~N}$ & (Chiang and Langway, 1978) \\
\hline Q-13 & Ross & -78.9568 & 179.9229 & $\mathrm{~N}$ & (Chiang and Langway, 1978) \\
\hline R7 & Brunt & -75.4 & -25.5 & $\mathrm{Y}$ & (Thomas, 1975) \\
\hline
\end{tabular}

Table S4: Sources for firn core data mapped in Figure 2.

\begin{tabular}{|l|l|l|l|l|}
\hline Shelf & Lat & Lon & Location notes & Reference \\
\hline Ross & -82.43 & 169.7 & & (Neal, 1979) \\
\hline Ross & - & - & $\begin{array}{l}\text { "Transition from valley glacier to ice } \\
\text { shelf" }\end{array}$ & (Robin et al., 1970) \\
\hline Larsen C & -68 & -60 & Near rifts in ice shelf & $\begin{array}{l}\text { (Smith, 1972; } \\
\text { Smith and Evans, 1972) }\end{array}$ \\
\hline Wordie & - & - & Towards edges of shelf & (Swithinbank, 1968) \\
\hline Wilkins & - & - & Map in Figure 2b & (Vaughan et al., 1993) \\
\hline McMurdo & - & - & Around boreholes in Figure 2e & (Grima et al., 2016) \\
\hline Brunt & -75.31 & -26.7 & $\begin{array}{l}\text { Attributed to basal marine ice by } \\
\text { Walford (1964) but to brine infiltration } \\
\text { by Swithinbank (1968) }\end{array}$ & $\begin{array}{l}\text { (Swithinbank, 1968; } \\
\text { Walford, 1964) }\end{array}$ \\
\hline
\end{tabular}

Table S5: Sources for radar observation of brine on Antarctic ice shelves, mapped in Figure 2. 


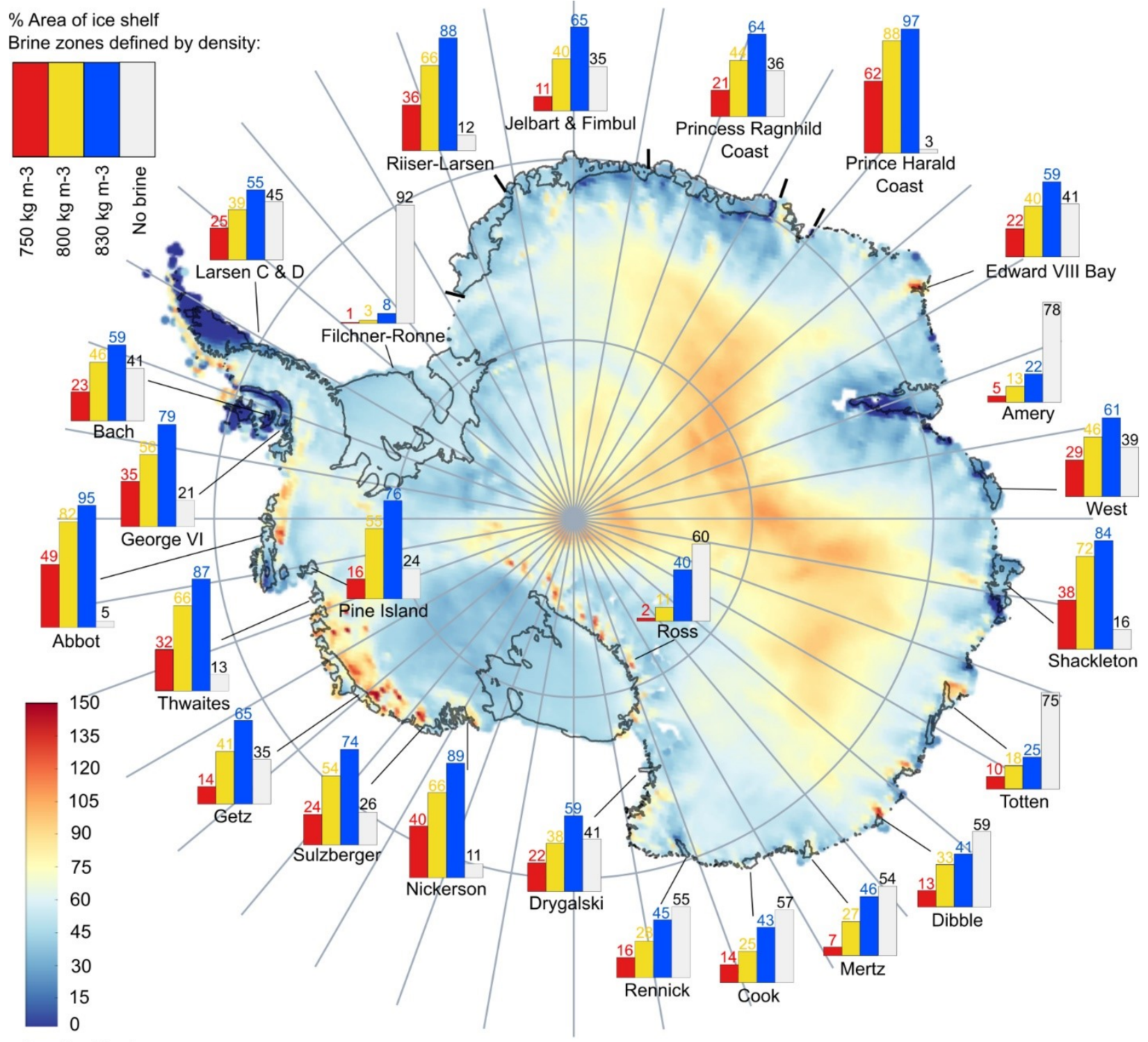

Depth of firn layer

Density $800 \mathrm{~kg} \mathrm{~m}^{-3}$

Figure S1: Depth of $800 \mathrm{~kg} \mathrm{~m}^{-3}$ firn layer. Data taken from firn densification model IMAUFDM (Ligtenberg et al., 2011). Bar charts show the percentage area of selected ice shelves covered by the brine zone, defined with a threshold for firn permeability of $750 \mathrm{~kg} \mathrm{~m}^{-3}$ (red), 800 $\mathrm{kg} \mathrm{m}^{-3}$ (yellow) and $830 \mathrm{~kg} \mathrm{~m}^{-3}$ (blue). Regions with particularly thick firn layers include Edward VIII Bay and Totten IS. 


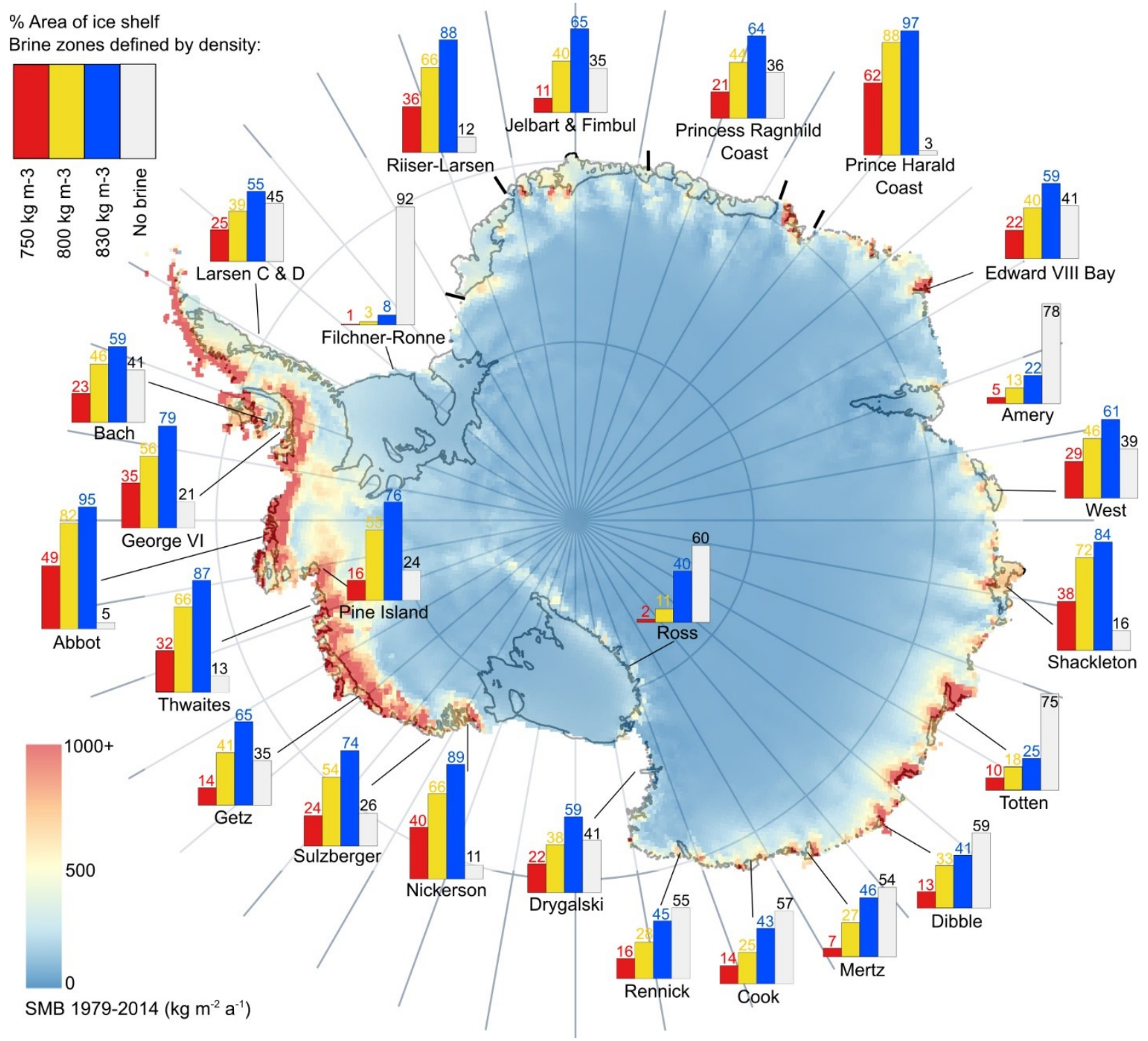

Figure S2: Surface mass balance in kg per square metre per annum (Van Wessem et al., 2014). Bar charts show the percentage area of selected ice shelves covered by the brine zone, defined with a threshold for firn permeability of $750 \mathrm{~kg} \mathrm{~m}^{-3}$ (red), $800 \mathrm{~kg} \mathrm{~m}^{-3}$ (yellow) and 830 $\mathrm{kg} \mathrm{m}^{-3}$ (blue). High accumulation rates coincide with shelves with large brine zones in the West Antarctic e.g. Abbot, Bach, Sulzberger \& Nickerson Ice Shelves as well as in other areas e.g. Edward VIII Bay. 


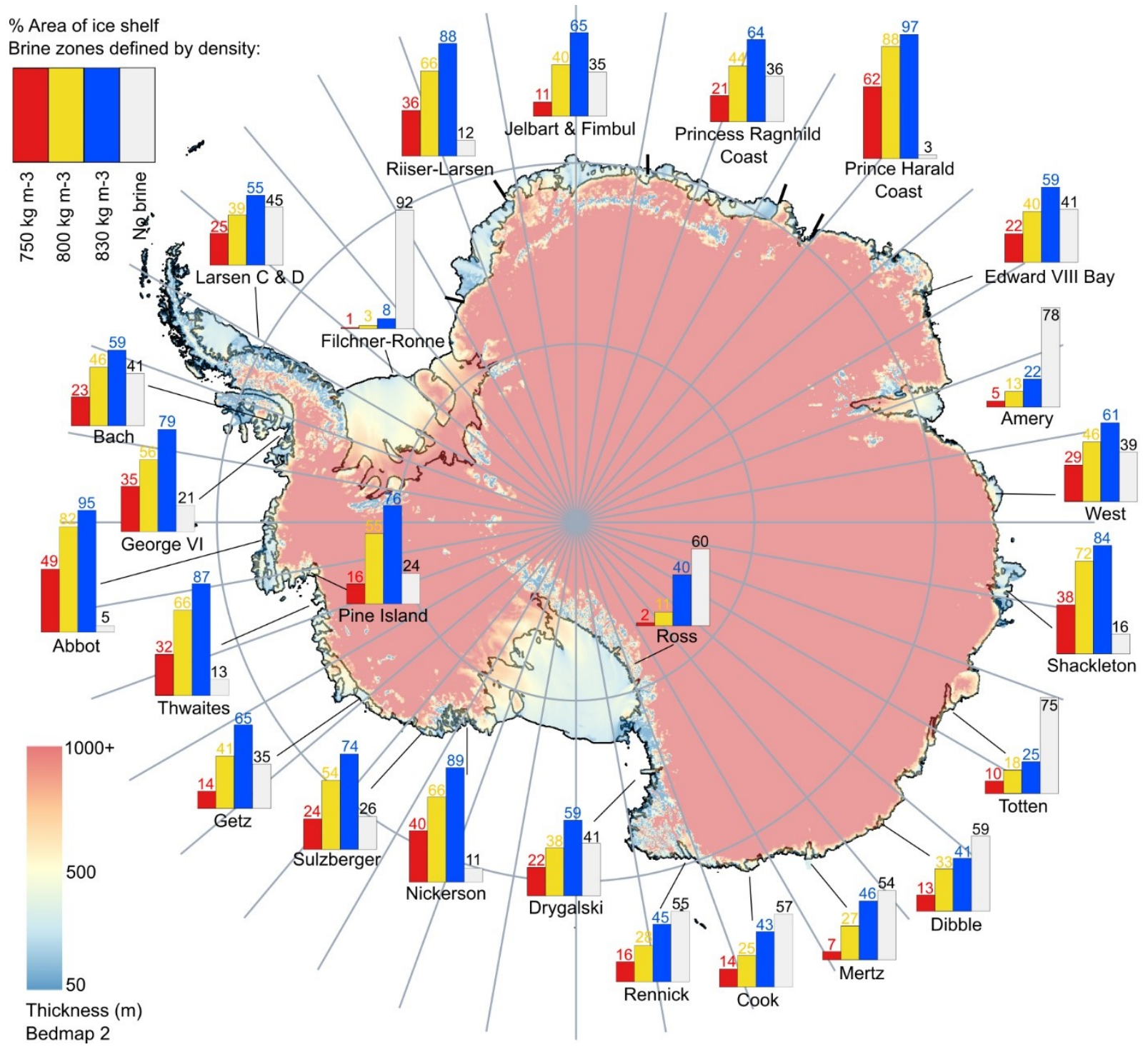

Figure S3: Ice thickness from Bedmap2 (Fretwell et al., 2013). Bar charts show the percentage area of selected ice shelves covered by the brine zone, defined with a threshold for firn permeability of $750 \mathrm{~kg} \mathrm{~m}^{-3}$ (red), $800 \mathrm{~kg} \mathrm{~m}^{-3}$ (yellow) and $830 \mathrm{~kg} \mathrm{~m}^{-3}$ (blue). Some ice shelves with a large brine zone coincide with areas of low ice thickness e.g. Riiser-Larsen and Princess Ragnhild regions and the Shackleton Ice Shelf. 


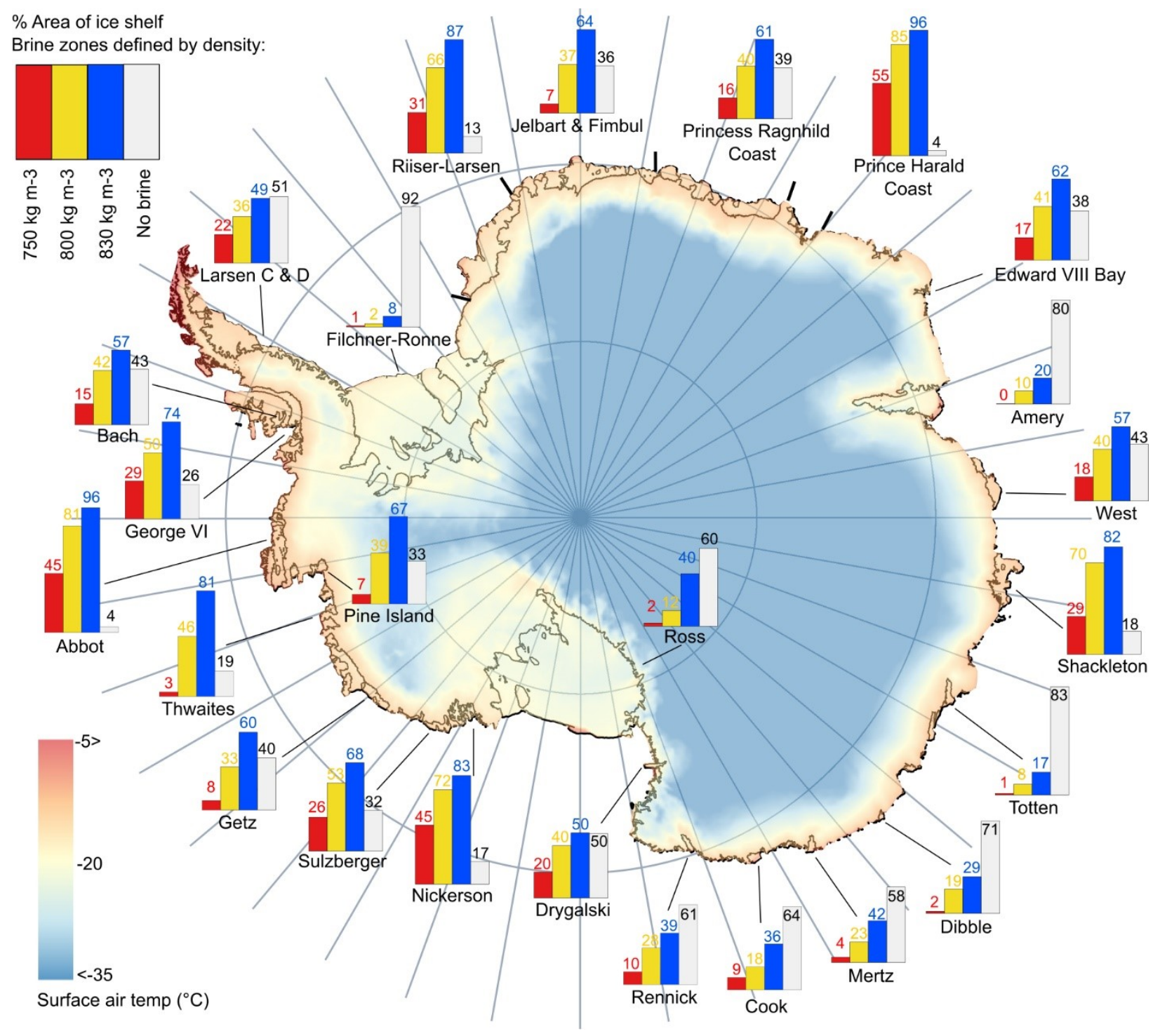

Figure S4: Surface air temperature from ALBMAPv1 Antarctic dataset (Le Brocq et al., 2010; Comiso, 2000). Annual mean temperatures averaged from 1982-2004. Bar charts show the percentage area of selected ice shelves covered by the brine zone, defined with a threshold for firn permeability of $750 \mathrm{~kg} \mathrm{~m}^{-3}$ (red), $800 \mathrm{~kg} \mathrm{~m}^{-3}$ (yellow) and $830 \mathrm{~kg} \mathrm{~m}^{-3}$ (blue). Very low annual mean temperatures will be reflected in the firn layer, and may cause freezing of seawater which will prevent its further infiltration. 


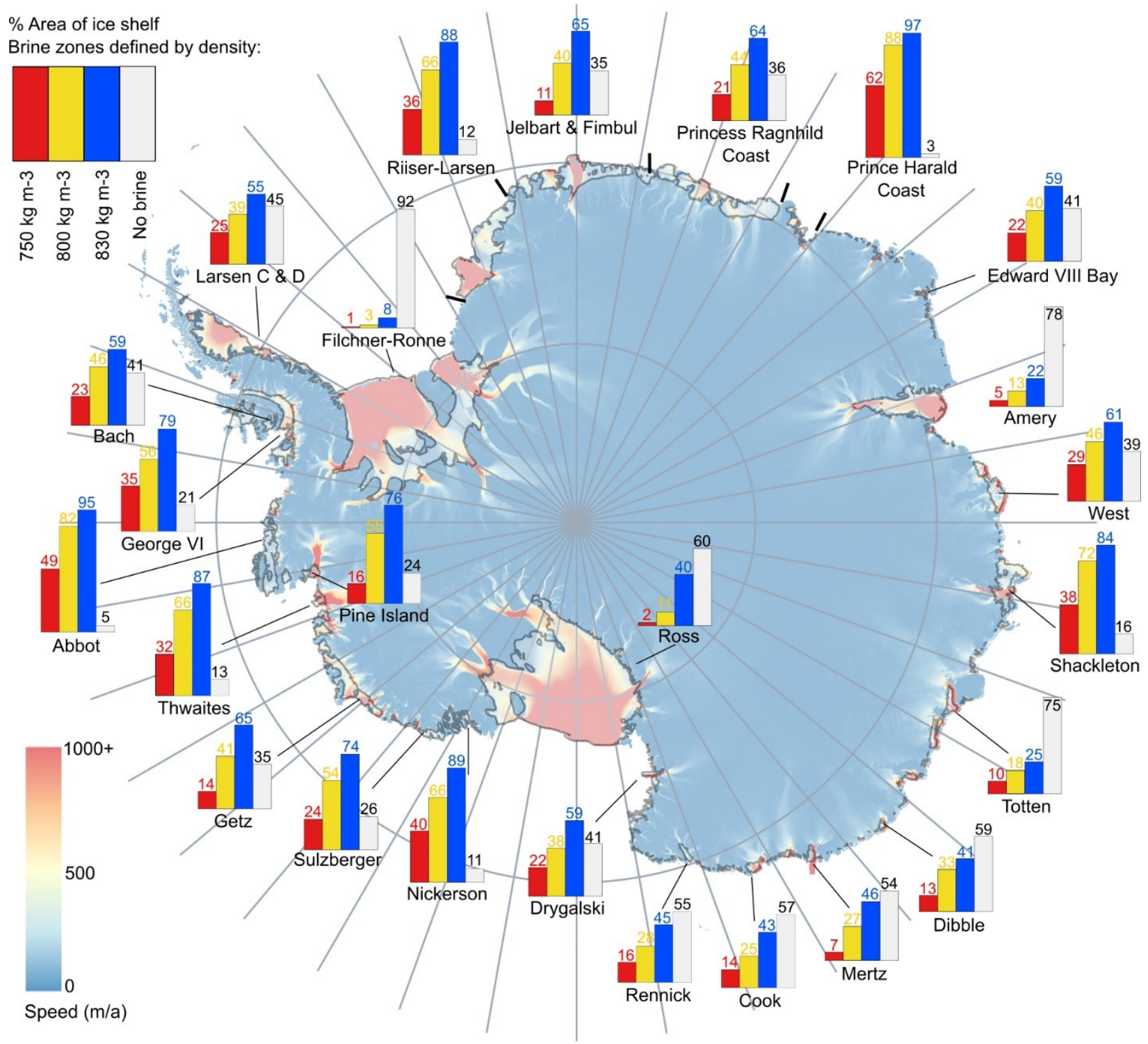

Figure S5: Ice speed from MEaSUREs InSAR-based Antarctica ice velocity map (Mouginot et al., 2017). Bar charts show the percentage area of selected ice shelves covered by the brine zone, defined with a threshold for firn permeability of $750 \mathrm{~kg} \mathrm{~m}^{-3}$ (red), $800 \mathrm{~kg} \mathrm{~m}^{-3}$ (yellow) and $830 \mathrm{~kg}$ $\mathrm{m}^{-3}$ (blue). Ice shelves with high flow speeds are less likely to be able to maintain a steady state area of liquid brine, if infiltration occurs from the calving front. 


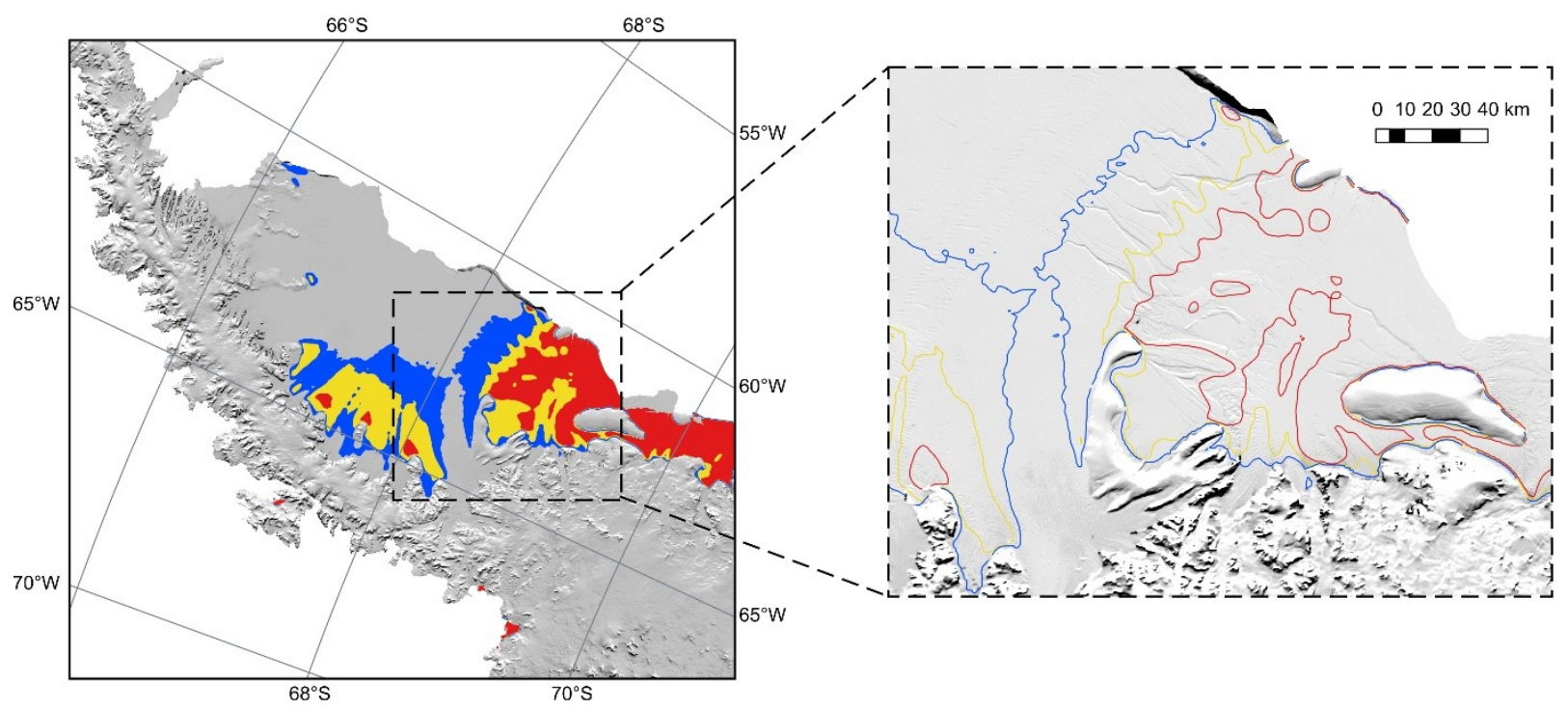

Figure S6: Brine zones on Larsen $\mathrm{C}$ ice shelf align with the source of rifts. Coloured areas show potential brine zones as described in Fig. 1. Background imagery is from MODIS Mosaic of Antarctica. The potential brine zone coincides with a region of rifting on the south side of the ice shelf. 


\section{References}

Le Brocq, A. M., Payne, A. J. and Vieli, A.: An improved Antarctic dataset for high resolution numerical ice sheet models (ALBMAP v1), Earth Syst. Sci. Data, 2(2), 247-260, 2010.

Budd, W. F., Corry, M. J. and Jacka, T. H.: Results from the Amery ice shelf project, Ann. Glaciol., 3(1), 36-41 [online] Available from: papers2://publication/uuid/83702729-1662-4B48B62F-D5C53EEBDEDD, 1982.

Chiang, E. and Langway, C. C.: Antarctic ice core recovery., Antarct. J. United States, 13(4), 59-61, 1978.

Comiso, J.: Variability and Trends in Antarctic Surface Temperatures from In Situ and Satellite Infrared Measurements, J. Clim., 13(10), 1674-1696, 2000.

Dubrovin, L. I.: Rassol v shel'fovom lednike Lazareva [Brine in the Lazarev Ice Shelf], Informatsionny Byulleten'Sovetkoj Antarkt. Ekspeditsii [Soviet Antarct. Exped. Inf. Bull., 22, 15-16, 1960.

Fretwell, P., Pritchard, H. D., Vaughan, D. G., Bamber, J. L., Barrand, N. E., Bell, R., Bianchi, C., Bingham, R. G., Blankenship, D. D., Casassa, G., Catania, G., Callens, D., Conway, H., Cook, A. J., Corr, H. F. J., Damaske, D., Damm, V., Ferraccioli, F., Forsberg, R., Fujita, S., Gim, Y., Gogineni, P., Griggs, J. A., Hindmarsh, R. C. A., Holmlund, P., Holt, J. W., Jacobel, R. W., Jenkins, A., Jokat, W., Jordan, T., King, E. C., Kohler, J., Krabill, W., Riger-Kusk, M., Langley, K. A., Leitchenkov, G., Leuschen, C., Luyendyk, B. P., Matsuoka, K., Mouginot, J., Nitsche, F. O., Nogi, Y., Nost, O. A., Popov, S. V., Rignot, E., Rippin, D. M., Rivera, A., Roberts, J., Ross, N., Siegert, M. J., Smith, A. M., Steinhage, D., Studinger, M., Sun, B., Tinto, B. K., Welch, B. C., Wilson, D., Young, D. A., Xiangbin, C. and Zirizzotti, A.: Bedmap2: Improved ice bed, surface and thickness datasets for Antarctica, Cryosphere, 7(1), 375-393, doi:10.5194/tc-7-3752013, 2013.

Gow, A. J.: Electrolytic conductivity of snow and glacier ice from Antarctica and Greenland, J. Geophys. Res., 73(12), 3643-3649, doi:10.1029/JB073i012p03643, 1968.

Graf, W., Moser, H., Oerter, H., Reinwarth, O. and Stichler, W.: Accumulation and ice-core studies on Filchner-Ronne Ice Shelf, Antarctica, Ann. Glaciol., 11, 23-31, 1988.

Graf, W., Moser, H., Reinwarth, O., Kipfstuhl, J., Oerter, H., Minikin, A. and Wagenbach, D.: Snow-accumulation rates and isotopic content $(2 \mathrm{H}, 3 \mathrm{H})$ of near-surface firn from the FilchnerRonne Ice Shelf, Antarctica., Ann. Glaciol., 20(1), 121-128, 1994.

Grima, C., Greenbaum, J. S., Lopez Garcia, E. J., Soderlund, K. M., Rosales, A., Blankenship, D. D. and Young, D. A.: Radar detection of the brine extent at McMurdo Ice Shelf, Antarctica, and its control by snow accumulation, Geophys. Res. Lett., 43(13), 7011-7018, doi:10.1002/2016GL069524, 2016.

Herron, M. M. and Langway, C. C.: Dating of Ross Ice Shelf cores by chemical analysis, J. Glaciol., 24(90), 345-357, 1979. 
Ligtenberg, S. R. M., Helsen, M. M. and Van Den Broeke, M. R.: An improved semi-empirical model for the densification of Antarctic firn, Cryosphere, 5(4), 809-819, doi:10.5194/tc-5-8092011, 2011.

Mouginot, J., Scheuchl, B. and Rignot, E.: MEaSUREs Annual Antarctic Ice Velocity Maps 2005-2016, Version 1., [online] Available from: doi: http://dx.doi.org/10.5067/9T4EPQXTJYW9., 2017.

Neal, C. S.: The dynamics of the Ross Ice Shelf revealed by radio echo-sounding, J. Glaciol., 24(90), 295-307, 1979.

Robin, G. de Q., Swithinbank, C. W. M. and Smith, B. M. E.: Radio echo exploration of the Antarctic ice sheet, Int. Assoc. Sci. Hydrol. Publ., 86, 97-115, doi:10.1017/S0032247400061143, 1970.

Schlosser, E. and Oerter, H.: Shallow firn cores from Neumayer, Ekstromisen, Antarctica: A comparison of accumulation rates and stable-isotope ratios, Ann. Glaciol., 35, 91-96, doi:10.3189/172756402781816915, 2002.

Schlosser, E., Oerter, H. and Graf, W.: Snow accumulation on Ekströmisen, Antarctica, 19801996, Berichte zur Polarforsch., 313, 1999.

Smith, B. M. E.: Airborne radio echo sounding of glaciers in the Antarctic Peninsula., 1972.

Smith, B. M. E. and Evans, S.: Radio echo sounding: absorption and scattering by water inclusion and ice lenses, J. Glaciol., 11(61), 133-146, 1972.

Swithinbank, C.: Radio echo sounding of Antarctic glaciers from light aircraft, Int. Assoc. Sci. Hydrol. Publ., 79, 405-414 [online] Available from: papers2://publication/uuid/5FA457C5A23F-4F4F-A21E-A9E6FC434058, 1968.

Thomas, R. H.: Liquid Brine in Ice Shelves, J. Glaciol., 14(70), 125-136, 1975.

Tongiorgi, E., Picciotto, E., De Breuck, W., Norling, T., Giot, J. and Pantanetti, F.: Deep drilling at Base Roi Baudouin, Dronning Maud Land, Antarctica., J. Glaciol., 4(31), 101-110, 1962.

Vaughan, D. G., Mantripp, R. D., Sievers, J. and Doake, C. S. M.: A synthesis of remote sensing data on Wilkins Ice Shelf, Antarctica, Ann. Glaciol., 17(1989), 211-218, 1993.

Walford, M. E. R.: Radio echo sounding through an ice shelf, Nature, 204(4956), 317-319, doi:10.1038/204317a0, 1964.

Van Wessem, J. M., Reijmer, C. H., Morlighem, M., Mouginot, J., Rignot, E., Medley, B., Joughin, I., Wouters, B., Depoorter, M. A., Bamber, J. L., Lenaerts, J. T. M., Van De Berg, W. J., Van Den Broeke, M. R. and Van Meijgaard, E.: Improved representation of East Antarctic surface mass balance in a regional atmospheric climate model, J. Glaciol., 60(222), 761-770, doi:10.3189/2014JoG14J051, 2014.

Zotikov, I. A., Zagorodnov, V. S. and Raikovsky, J. V.: Core Drilling Through the Ross Ice Shelf (Antarctica) Confirmed Basal Freezing., Science (80-. )., 207(March), 1463-5, doi:10.1126/science.207.4438.1463, 1980. 